\title{
APLICAÇÃO DAS METODOLOGIAS FMEA E GUT NA PRESTAÇÃO DO SERVIÇO AUTOMOTIVO DE GEOMETRIA A LASER
}

\author{
APPLICATION OF FMEA AND METHODOLOGIES IN SERVICE \\ DELIVERY GUT AUTOMOTIVE LASER GEOMETRY
}

\author{
Data de submissão: 19-06-2012 \\ Aceite: 04-12-2012 \\ Priscila Cembranel ${ }^{1}$ \\ Luis Felipe Dias Lopes ${ }^{2}$
}

\section{RESUMO}

O serviço automotivo de geometria a laser é responsável pelo alinhamento das rodas, da direção e do câmber e tem como objetivo ajustar as rodas para que o pneu tenha o mínimo atrito possível com o solo. Selecionou-se o serviço de geometria a laser devido a sua grande procura na empresa em estudo (verificado por meio dos relatórios de vendas e ordens de serviços), no que diz respeito ao volume de vendas. O estudo teve como objetivo a elaboração do fluxograma de processos, a identificação das falhas nos processos sugerindo a implementação de ações para a correção das inconformidades. Como metodologia utilizou-se a abordagem quantitativa, metodologia descritiva e como técnica o acompanhamento in loco, de forma a identificar todas as etapas envolvidas no processo de prestação do serviço. Para a identificação das falhas utilizou-se a metodologia FMEA, que foram avaliadas utilizando-se os critérios da metodologia GUT. As principais falhas elencadas foram: o alto índice de retrabalho, a qualificação do geometrista, a necessidade de atualização do manual dos padrões e medidas dos veículos para a geometria, a necessidade de acompanhamento do geometrista junto ao cliente nos testes do veículo e a verificação e aferição da máquina de geometria. Através da metodologia GUT elencou-se como prioridade o acompanhamento do geometrista junto ao cliente nos testes do veículo e a aferição do equipamento de geometria, o que impactou diretamente no índice de retrabalho.

Palavras chave: Geometria a laser, metodologia FMEA, metodologia GUT, resolução de problemas, priorização.

\footnotetext{
1 Possui graduação em Administração pela Sociedade Educacional Três de Maio, SETREM, especialização em MBA Gestão de Pessoas e Desenvolvimento de Talento pela Sociedade Educacional Três de Maio, SETREM, mestrado em Engenharia de Produção pela Universidade Federal de Santa Maria, UFSM e doutorado em andamento em Administração e Turismo pela Universidade do Vale do Itajaí, UNIVALI. Biguaçu. Santa Catarina. Brasil. E-mail: priscila_cembranel@yahoo.com.br

2 Possui graduação em Matemática Licenciatura Plena pela Faculdade Imaculada Conceição, FIC, especialização em Estatística e Modelagem Quantitativa pela Universidade Federal de Santa Maria, UFSM, mestrado em Engenharia de Produção pela Universidade Federal de Santa Maria, UFSM e doutorado em Engenharia de Produção pela Universidade Federal de Santa Catarina, UFSC. Atualmente coordena 20 projetos de pesquisa. Coordenador da Região Central do RS do Projeto Negócio a Negócio do SEBRAE. Membro do Comitê de Assessores na área de Tecnologia e Inovação da FAPERGS. Membro do Comitê de Avaliadores da FAPEMA. Membro do Comitê de Avaliadores da FAPESPA, Membro do Comitê de Avaliadores da FAPESC e Membro do Comitê Científico da CAPES na área de Engenharia de Produção. Santa Maria. Rio Grande do Sul. Brasil. E-mail: Iflopes67@yahoo.com.br
} 


\begin{abstract}
The automotive service geometry laser is responsible for wheel alignment, steering and camber and aims to adjust the wheels so the tire has the least possible friction with the ground. We selected the service Geometry laser due to its high demand in the company under study (verified by the sales reports and service orders), with respect to sales volume. The study aimed at the development of process flowchart, identifying failures in suggesting and implementing actions to correct the non-conformities. The methodology we used the quantitative approach, methodology and descriptive as technical monitoring in situ, in order to identify all steps involved in the process of providing the service. For the identification of faults used the FMEA method was evaluated using criteria from methodology GUT. The main faults listed were: the high rate of rework, the qualification of contractor geometry, the need to update the manual of standards and measures for the vehicle geometry, the need for monitoring contractor geometry with the client in the vehicle testing and calibration and verification machine geometry. Through the methodology GUT has listed as priority monitoring the contractor geometry with the client in the vehicle testing and measuring equipment geometry, which directly impacted the index rework.
\end{abstract}

Keywords: laser geometry, FMEA methodology, methodology GUT, problem solving, prioritization.

\title{
1 INTRODUÇÃO
}

O presente estudo foi realizado em uma organização prestadora de serviços automotivos na região do Vale dos Sinos, no Rio Grande do Sul. Os frequentes problemas relacionados ao retrabalho na prestação do serviço de geometria a laser dificultam a fidelização e a prospecção de novos clientes. A aplicabilidade das metodologias foi demonstrada principalmente através de tabelas, contribuindo no controle e diagnóstico dos entraves relacionados ao processo, e a priorização de determinadas ações em relação à necessidade de melhorias da prestação do serviço de geometria a laser. Após a implementação das metodologias o controle passou a ser registrado, padronizado e contínuo.

O principal motivo para serem implementadas as metodologias FMEA (do inglês Failure Mode and Effect Analysis) e GUT (Gravidade, Urgência e Tendência) foi a falta de controle e a inexistência do mapeamento das etapas da prestação do serviço de geometria a laser. Dessa forma, a utilização das tabelas padronizadas tornou-se uma possibilidade de controle das etapas facilitando a identificação de falhas e priorizando a resolução das que causam maior impacto à prestação do serviço.

\section{METODOLOGIA}

O serviço de geometria é responsável pelo alinhamento das rodas, da direção e do câmber ajustando as rodas para que o pneu tenha o mínimo atrito possível com o solo.

Selecionou-se o serviço de geometria por ser um dos serviços mais procurados (verificandose através dos relatórios de vendas e ordens de serviços) no que diz respeito ao volume de vendas.

O estudo teve como objetivo a elaboração do fluxograma de processos, a identificação das falhas nos processos e a sugestão e a implementação de ações para a correção dos problemas identificados. Através de uma abordagem quantitativa, utilizou-se como metodologia a pesquisa descritiva tendo em vista o envolvimento de técnicas padronizadas de coleta de dados, através dos formulários utilizados pelas metodologias FMEA e GUT. As técnicas utilizadas foram: a pesquisa bibliográfica a fim de levantar os conhecimentos já existentes sobre as metodologias e sua aplicabilidade e o acompanhamento in loco, como forma de identificar todas as etapas envol- 
vidas no processo de prestação do serviço de geometria a laser. Para a identificação das falhas, utilizou-se a metodologia FMEA. Por fim, as falhas levantadas foram avaliadas utilizando-se os critérios da metodologia GUT.

\section{CONSIDERAÇÕES PRELIMINARES}

A suspensão dianteira de um veículo é um sistema maleável criado para absorver os impactos da melhor forma possível. Quando submetidos às irregularidades da malha rodoviária, os pontos de fixação da suspensão perdem a exata localização da fixação original (feita através de parafusos ajustáveis). (ABRAPNEUS, 2009)

A geometria serve para regular os pontos de fixação fazendo com que as rodas fiquem centralizadas formando um retângulo. As máquinas de geometria jamais regulariam os pontos de fixação de forma precisa (como quando os veículos saem das fábricas), por isso, existe uma margem de erro para os graus e milímetros especificados pelas montadoras. Desse modo, o serviço de geometria, por não ser algo preciso, possui índices de retrabalho que, dependendo das circunstâncias podem ser considerados altos. (ABRAPNEUS, 2009).

Eficiência e eficácia tornaram-se fundamentais para a sobrevivência das organizações que enfrentam a necessidade de diminuição de custos na prestação de serviços e a exigência de padrões de qualidade cada vez mais rígidos solicitados pelos usuários.

A metodologia de Análise do Tipo e Efeito de Falha, (do inglês Failure Mode and Effect Analysis), é uma ferramenta que procura evitar que ocorram falhas no projeto do produto ou processo, através da análise das falhas possíveis e da sugestão de melhorias buscando aumentar a confiabilidade de processos e materiais.

A metodologia GUT (Gravidade, Urgência e Tendência), é uma ferramenta que busca avaliar e atribuir valores aos aspectos observados, segundo os parâmetros de gravidade, urgência e tendência priorizando a resolução dos problemas que apresentarem maior pontuação.

\section{ANÁLISE DO TIPO E EFEITO DE FALHA (FMEA)}

Stamatis (2003) define FMEA (Análise dos Modos e Efeitos das Falhas) como um método de análise de produtos ou processos usados para identificar todos os possíveis modos potenciais de falha e determinar o efeito de cada um sobre o desempenho do sistema (produto ou processo), mediante um raciocínio basicamente dedutivo (não exige cálculos sofisticados). É, portanto, um método analítico padronizado para detectar e eliminar problemas potenciais de forma sistemática e completa.

Puente (2002) salienta que o desenvolvimento da Análise dos Modos e Efeitos das FaIhas (FMEA), consiste em: reconhecer e avaliar as possíveis falhas e seus efeitos, encontrar ações que possam minimizar ou eliminar potenciais falhas, e documentar os processos. Procura-se determinar modos de falhas dos componentes mais simples, as suas causas e de que maneira eles afetam os níveis superiores da produção. Para Fernandes e Rebelatto (2006), existem três tipos de FMEA: de produto, de processo e de sistema. No FMEA de produto, as causas de falha são as do projeto do produto (mau dimensionamento, má especificação de material, entre outros), na FMEA de processo as causas das falhas serão devido a algum problema do processo de fabricação e a FMEA de sistema possui ligação com a percepção do cliente em relação à organização focando as falhas do sistema em relação às funcionalidades organizacionais.

A metodologia FMEA é uma forma sistemática de relacionar informações sobre falhas dos produtos/processos, melhorando o conhecimento dos problemas sendo capaz de apontar 
ações de melhoria no projeto, de diminuição de custos por meio da prevenção de ocorrência de falhas e de prevenção de falhas, como salientam Zambrano e Martins (2007).

Para Fernandes (2005), o preenchimento de dados deve ser realizado pelo responsável pela aplicação, devendo conter: a descrição dos objetivos e abrangência da análise, qual produto ou processo será analisado, a formação dos grupos de trabalho, que deve ser pequeno (entre 4 a 6 pessoas) e com pessoas de diversas áreas. Deve ocorrer também o planejamento das reuniões com antecedência sendo primordial a aceitação de todos os participantes e a preparação da documentação.

A análise das fases em potencial deve ser realizada pelo grupo de trabalho, que discute e preenche o formulário FMEA, definindo: a função e característica do produto/processo, tipo de faIha potencial para cada função, efeito do tipo de falha, causa possível da falha e os controles atuais.

Após, há necessidade de avaliar os riscos através dos índices de severidade ( $\mathrm{S}$ ), ocorrência (O) e detecção (D) para cada causa de falha, de acordo com critérios definidos pela empresa. Depois, são calculados os coeficientes de prioridade de risco (R), por meio da multiplicação dos outros três índices. Em seguida, há a fase de melhoria onde é possível listar todas as ações que podem ser realizadas para diminuir os riscos. Para Rosa e Garrafa (2009), as medidas de avaliação da probabilidade de ocorrência de falhas, a prevenção total ao tipo de falha ou suas causas, as formas de coibir a ocorrência de falhas, as limitações do efeito do tipo de falha, o aumento da probabilidade de detecção do tipo ou da causa de falha podem ser ações adotadas para diminuição dos riscos, quando consideradas viáveis.

Para a elaboração de uma análise via FMEA, sugere Helman (1995, p. 162), seguir os passos indicados:

10 - Definir a Equipe Responsável pela Execução.

20 - Definir os Itens do Sistema que serão Considerados.

3o - Preparação Prévia e Coleta de Dados.

40 - Análise Preliminar dos Itens Considerados.

50 - Identificação dos Modos de Falha e seus Efeitos.

6ㅇ - Identificação das Causas das Falhas.

70 - Identificação dos Controles Atuais de Detecção das Falhas.

8 - Determinação dos Índices de criticalidade (O corrência, G ravidade, D etecção e R isco).

9o - Análise das Recomendações.

10 - - Revisão dos Procedimentos.

110 - Preenchimento dos Formulários de FMEA, a partir, das Listas de Verificação.

12o - Reflexão sobre o Processo. (HELMAN, 1995, p. 162).

Observa-se que, uma vez que realizada a análise FMEA para produto ou processo, esta deve ser novamente realizada quando ocorrerem ou não alterações nesse produto ou processo com a finalidade de confrontar as falhas já corrigidas e verificar potenciais falhas ainda não diagnosticadas.

\section{GRAVIDADE, URGÊENCIA E TENDÊNCIA (GUT)}

De acordo com Behr e Estabel (2008), a matriz GUT - Gravidade, Urgência e Tendência deve ser um meio de priorizar ações na gestão. Consiste em analisar a gravidade ou o impacto do problema nas operações e das pessoas envolvidas, a urgência ou a brevidade necessária para a resolução dos problemas e a tendência ou apresentação de melhora ou piora do problema. Para Nascimento et. al. (2010), o estabelecimento de metas é importante para a visualização do nível de melhoria que pode ser incorporado ao processo. 
Tabela 1 - Metodologia GUT.

\begin{tabular}{|c|c|c|c|c|c|}
\hline \multicolumn{2}{|r|}{ Gravidade } & \multicolumn{2}{|r|}{ Urgência } & \multicolumn{2}{|r|}{ Tendência } \\
\hline 1 & $\begin{array}{l}\text { Sem gravidade. Dano } \\
\text { mínimo }\end{array}$ & 1 & $\begin{array}{l}\text { longuíssimo prazo (dois ou } \\
\text { mais meses) }\end{array}$ & 1 & Sem tendência de piorar \\
\hline 2 & $\begin{array}{l}\text { Pouco grave. Dano } \\
\text { leve }\end{array}$ & 2 & $\begin{array}{c}\text { Pouco Urgente. Longo prazo } \\
\text { (um mês) }\end{array}$ & 2 & Vai piorar em longo prazo \\
\hline 3 & Grave. Dano regular & 3 & $\begin{array}{l}\text { Urgente. Prazo médio (uma } \\
\text { quinzena) }\end{array}$ & 3 & $\begin{array}{l}\text { Vai piorar em médio } \\
\text { prazo }\end{array}$ \\
\hline 4 & $\begin{array}{c}\text { Muito Grave. Grande } \\
\text { dano }\end{array}$ & 4 & $\begin{array}{l}\text { Muito urgente. Curto prazo } \\
\text { (uma semana) }\end{array}$ & 4 & Vai piorar em curto prazo \\
\hline 5 & $\begin{array}{c}\text { Extremamente grave. } \\
\text { Dano gravíssimo }\end{array}$ & 5 & $\begin{array}{l}\text { Extremamente urgente. Ime- } \\
\text { diatamente (está ocorrendo) }\end{array}$ & 5 & $\begin{array}{l}\text { Se não for resolvido, pio- } \\
\text { ra imediatamente }\end{array}$ \\
\hline
\end{tabular}

Fonte: Adaptado de Behr e Estabel (2008).

Cada problema é ponderado através de critérios numerados que vão de um a cinco, tendo como base para ponderação a Tabela 1. Após a ponderação, somam-se na horizontal os valores de cada problema e pelo total obtido eles se hierarquizam. (BEHR e ESTABEL, 2008)

Para Oliveira (1999), a metodologia GUT avalia cada fator considerado na análise da situação à luz dos critérios de gravidade, urgência e tendência. Mesmo sendo uma metodologia desenvolvida para a fixação de prioridades no diagnóstico estratégico, ela pode também ser aplicada para identificar problemas existentes colaborando com a postura estratégica organizacional. Seu objetivo é orientar os processos de tomada de decisão e a resolução de problemas. Após o levantamento das causas, a matriz GUT permite quantificar cada causa de acordo com a gravidade, urgência e tendência. (CUNHA, 2010)

\section{ANÁLISE E DISCUSSÃO DOS RESULTADOS}

A geometria é o alinhamento das rodas, da direção e do câmber ajustando as rodas para que o pneu tenha o mínimo atrito possível com o solo.

$\mathrm{Na}$ empresa estudada, o processo de geometria a laser acontece em três etapas. Inicialmente, o carro é colocado na rampa de geometria a laser podendo ser elevado ou não. Em seguida, o projetor a laser é fixado na roda. A primeira etapa consiste na verificação dos graus do câmber no painel de geometria (varia de acordo com o modelo do carro), não estando dentro das medidas aceitáveis realiza-se a correção através da fixação do ciborgue automotivo na torre do amortecedor. Na segunda etapa, com o projetor ainda fixado na roda a convergência, é verificada através da medição e leitura dos milímetros ou graus relativos a angulação da roda, através de um facho de raio laser que sai do projetor; esse laser é refletido em um espelho e retorna no sistema de régua do projetor fornecendo a medida. Não estando dentro dos padrões, a correção é realizada através da barra axial com uma chave fixa (de boca). A última etapa é o alinhamento da direção, o projetor ainda fixado na roda dianteira é virado em 180 graus direcionando os fachos de laser em uma régua que é fixada do lado externo nas rodas traseiras. Feita a verificação e detectando-se alguma inconformidade nos padrões (em milímetros) a correção é realizada através da barra axial com uma chave fixa (de boca). 
Figura 1 - Fluxograma de processos do serviço de Geometria a Laser.

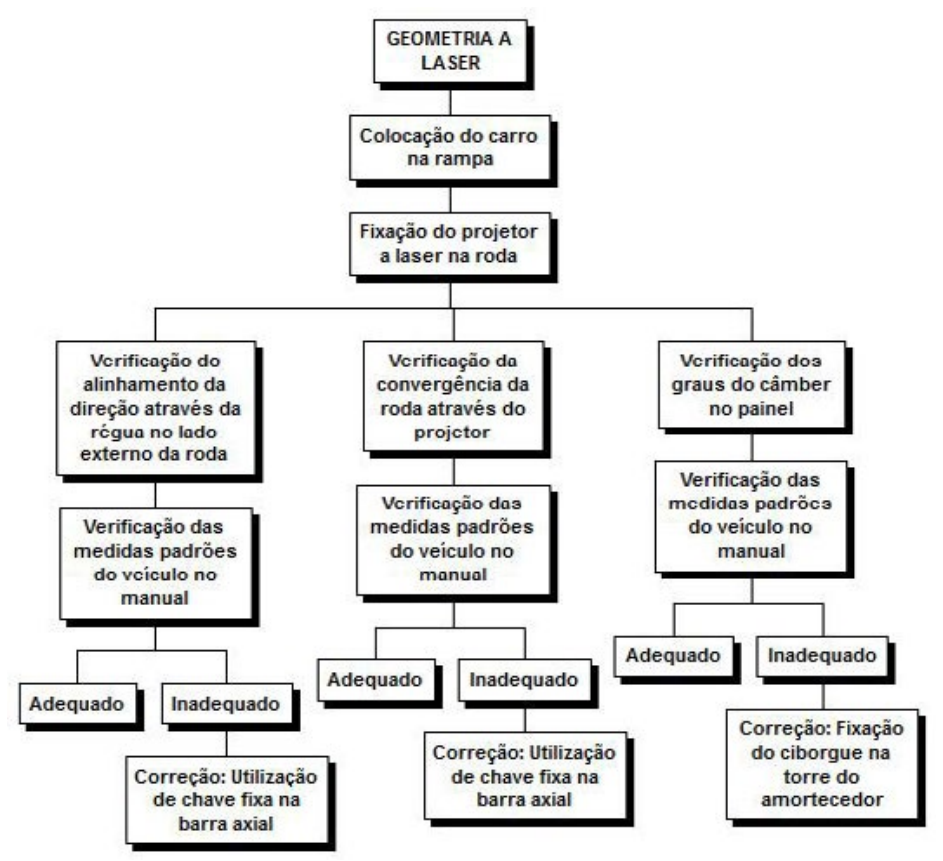

Quando o serviço prestado é insatisfatório, os principais aspectos observados no veículo relacionam-se à convergência (quando o carro tende a puxar para um determinado lado), causando o desgaste do pneu e podendo aumentar o consumo de combustível. Outro aspecto, que pode ser observado, é a direção estar desalinhada quando andando em linha reta. Neste caso, não há grandes danos para o veículo. Para a empresa, estes resultados geram a insatisfação dos clientes e o retrabalho. As principais causas elencadas para as falhas são: falta de conhecimento ou verificação das medidas adequadas para cada carro e a desatenção ou falta de capacitação do geometrista.

A metodologia FMEA (Análise dos modos e efeitos de falhas), pressupõe o controle dos critérios de severidade, ocorrência e detecção. Para a análise dos resultados utilizou-se os índices, o critério e as especificações de cada critério de acordo com o número representado. A severidade foi analisada de acordo com a Tabela 2, a ocorrência de acordo com a Tabela 3 e a deteç̧ão de acordo com a Tabela 4.

Tabela 2 - Critério de risco: Severidade.

\begin{tabular}{ccl}
\hline ÍNDICE & SEVERIDADE & CRITÉRIO \\
\hline 1 & Mínima & O cliente quase não percebe a ocorrência da falha. \\
2 & Pequena & Pequena falha no desempenho e pequeno descontentamento do cliente. \\
3 & Moderada & Falhas significativas no desempenho e descontentamento do cliente. \\
4 & Alta & Sistema deixa de funcionar e grande descontentamento do cliente. \\
5 & Muito alta & Problemas de segurança. \\
\hline
\end{tabular}

Fonte: Os autores (2012).

Tabela 3 - Critério de risco: Ocorrência.

\begin{tabular}{ccc}
\hline IDICE & OCORRENCIA & PROPORCÁO \\
\hline 1 & Remota & $1: 50$ \\
2 & Pequena & $1: 40$ \\
3 & Moderada & $1: 30$ \\
4 & Alta & $1: 20$ \\
5 & Muito alta & $1: 10$ \\
\hline Fonte: Os autores (2012). & &
\end{tabular}


Tabela 4 - Critério de risco: Detecção.

\begin{tabular}{ccc}
\hline ÍNDICE & DETECÇÃO & PROPORÇÃO \\
\hline 1 & Muito grande & Certamente será detectado. \\
2 & Grande & Grande chance de ser detectado. \\
3 & Moderada & Provavelmente será detectado. \\
4 & Pequena & Provavelmente não será detectado. \\
5 & Muito pequena & Certamente não será detectado. \\
\hline
\end{tabular}

Fonte: Os autores (2012).

O diagnóstico apontou que, além da desatenção em relação ao manual fornecido pela empresa (para a verificação das medidas aceitáveis para cada modelo de veículo) e a falta de capacitação do geometrista, também pôde ser verificada a falta de aferição do equipamento de geometria que impacta diretamente nos resultados. Dos clientes agendados para refazerem o serviço, metade solicitou o acompanhamento do geometrista para a realização do teste do carro (uma das soluções adotadas permanentemente após a aplicação da metodologia FMEA). Para a resolução dos problemas observados, elencou-se a necessidade de qualificação do geometrista (fornecida pela empresa), a atualização do manual que contém os padrões e medidas de cada veículo e a realização dos testes de verificação junto ao cliente quando possível.

Figura 2 - Fases da aplicação da metodologia FMEA no processo de geometria a laser.

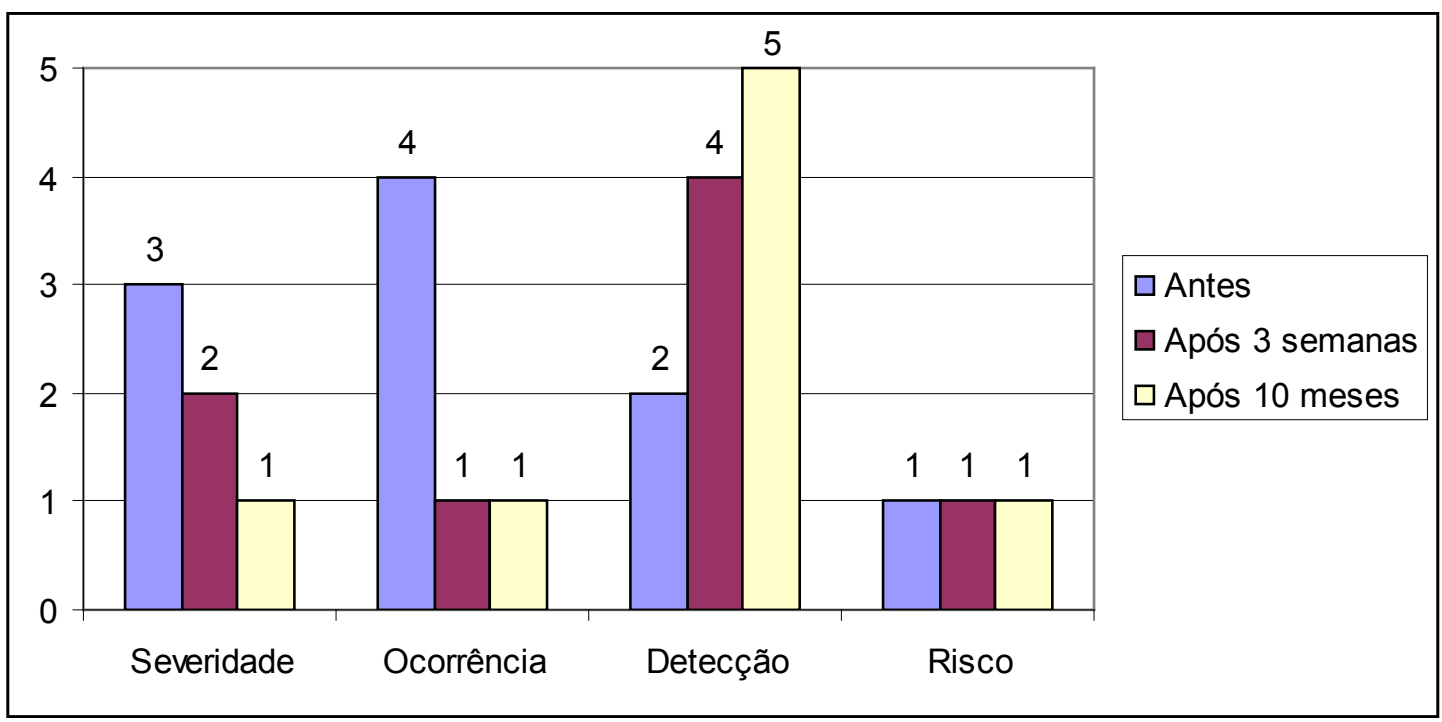

De acordo com a Figura 2, inicialmente, o índice de severidade foi considerado moderado, pois as falhas afetavam significativamente o desempenho do processo, o que gerava o descontentamento do cliente (índice 3). Após a aplicação da metodologia, esse índice passou a ser considerado pequeno, pois o cliente raramente percebia a ocorrência da falha (índice 2). Dez meses depois, verificou-se que o cliente praticamente não percebia a ocorrência da falha (índice 1). A ocorrência de falhas antes da aplicação do FMEA foi considerada alta, na proporção de 1:20 (índice 4). Após a aplicação da metodologia, a ocorrência passou a ser considerada remota, na proporção 1:50 (índice 1), mantendo o mesmo índice após os dez meses de utilização das metodologias FMEA e GUT. Antes da aplicação da metodologia, o critério de detecção foi considerado grande, pois certamente o cliente conseguiu detectar a falha (índice 2). Após a aplicação, a detecção passou a ser pequena, já que o cliente provavelmente não detectarou nenhuma falha (índice 4). Na última verificação, dez 
meses após a introdução da metodologia, a deteç̧ão passou a ser muito pequena, pois o cliente certamente não detectou a falha (índice 5). Os riscos de a situação repetir-se, antes da aplicação da metodologia, manteve-se o mesmo depois da aplicação da metodologia (índice 1).

A metodologia FMEA detectou as falhas nas etapas do serviço fornecendo sugestões de melhorias. Dentre as medidas a serem tomadas pode-se citar: a qualificação do geometrista, o índice de retrabalho, o acompanhamento do geometrista no teste do veículo e a aferição da máquina de geometria. A priorização das ações elencadas através da metodologia FMEA foi realizada através da metodologia GUT (Gravidade, Urgência e Tendência), como pode ser observado na Tabela 5. Considerando-se a gravidade como a intensidade dos danos que um problema pode causar, caso nenhuma medida seja tomada, a urgência relaciona-se ao tempo até que os danos ou resultados indesejáveis possam ser percebidos, caso nada seja feito em relação ao problema. Já a tendência considera o desenvolvimento do problema, caso nada seja feito para sua resolução.

Tabela 5 - Aplicação da Metodologia GUT após 3 semanas e após 10 meses.

\begin{tabular}{cccccccccc}
\hline \multirow{2}{*}{ PROBLEMA } & \multicolumn{3}{c}{ G } & \multicolumn{3}{c}{ U } & \multicolumn{1}{c}{ T } & \multicolumn{2}{c}{ TOTAL } \\
\cline { 2 - 9 } & Antes & Depois & Antes & Depois & Antes & Depois & Antes & Depois \\
\hline Qualificação do geometrista & 5 & 4 & 3 & 2 & 2 & 1 & 10 & 7 \\
Retrabalho & 3 & 2 & 4 & 2 & 1 & 1 & 8 & 5 \\
Acompanhamento do cliente nos testes & 3 & 2 & 3 & 2 & 2 & 1 & 8 & 5 \\
Aferição do equipamento de geometria & 5 & 1 & 4 & 2 & 5 & 3 & 14 & 6 \\
Fonte: Os autores (2012). & & & & & & & & \\
\hline
\end{tabular}

Conforme a Tabela 5 pode-se observar que a qualificação do geometrista é considerada um dano gravíssimo, com necessidade de resolução em médio prazo (no máximo quinze dias), podendo esta piorar em longo prazo caso nenhuma medida seja tomada. $O$ retrabalho é considerado um dano grave a ser resolvido em curto prazo (uma semana), sendo que se nenhuma medida for tomada a tendência é que a ocorrência aumente. $\mathrm{O}$ acompanhamento dos clientes nos testes foi considerado um aspecto grave e urgente que pode piorar no longo prazo. A aferição do equipamento de geometria é considerada um dano gravíssimo, com urgência considerada de curto prazo (uma semana), sendo que a tendência é piorar imediatamente, caso nenhuma medida seja tomada. Na avaliação realizada após o intervalo de dez meses, a qualificação do geometrista passou a ser considerado um problema causador de grandes danos que deve ser solucionado no longo prazo (máximo um mês), cuja tendência é o desaparecimento. $O$ retrabalho e o acompanhamento do cliente nos testes foram considerados causadores de danos leves e passíveis de resolução no longo prazo (um mês), com tendência ao desaparecimento. Finalmente, a aferição do equipamento de geometria com as verificações regulares, passou a ser considerado um dano mínimo a ser resolvido no longo prazo (um mês), mas com tendência a permanecer. 
Figura 3 - Matriz Gravidade e Urgência para priorização de ações.

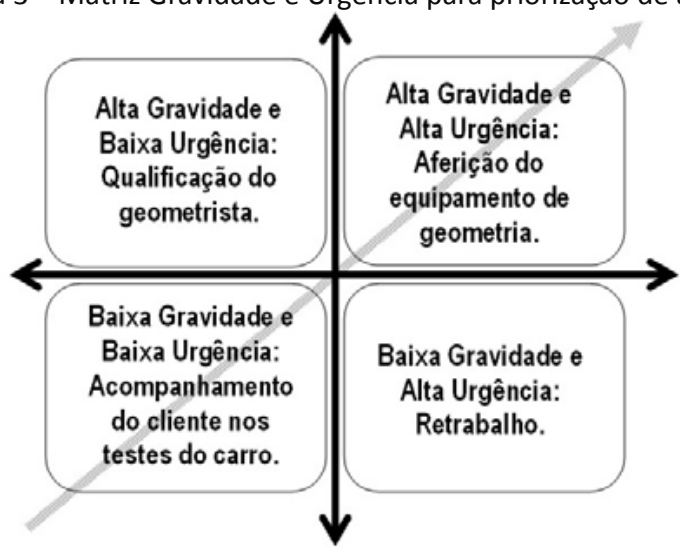

Os problemas foram priorizados de acordo com a combinação dos critérios gravidade e urgência (conforme Figura 3). Como houve a contratação de profissionais com maior nível de qualificação, após a primeira análise realizada através da metodologia FMEA, os esforços concentraram-se no acompanhamento do cliente, quando possível, nos testes do veículo após a prestação do serviço de geometria. Percebeu-se que a alta urgência verificada em relação ao retrabalho diminuiria consideravelmente se o geometrista fosse responsável pela realização dos testes no veículo após o serviço e se, além disso, o cliente estivesse presente neste momento. O principal problema elencado pela metodologia GUT foi a aferição do equipamento de geometria. Diante disso, a organização optou por agendar uma verificação quinzenal e se necessária uma aferição semanal, realizada pelo próprio operador (que recebeu treinamento).

\section{CONSIDERAÇÕES FINAIS, LIMITAÇÕES E RECOMENDAÇÕES}

A geometria exata ou dentro dos padrões das montadoras garante a estabilidade do carro diminuindo o consumo de combustível e o desgaste dos pneus. No caso da empresa em estudo, o alto índice de retrabalho em relação aos serviços de geometria prestados estava gerando a insatisfação e até a perda de clientes.

Mesmo depois da aplicação das metodologias FMEA e GUT durante dez meses e da organização ter se comprometido a dar continuidade a estes registros aplicando as ações corretivas quando necessário, existem limitações no estudo em relação ao no serviço de geometria a laser. Sabe-se que, existem falhas que ainda persistem, mesmo diante dos esforços frente aos índices de retrabalho (e a considerável diminuição destes, após a aplicação do presente estudo), não existem garantias de que a aplicação contínua das metodologias FMEA e GUT tornem os índices de retrabalho e redução de falhas inexpressivas ou inexistentes.

A metodologia FMEA foi utilizada para descobrir as falhas do serviço prestado, inicialmente foi necessário mapear suas etapas e processos. Diante da primeira verificação, a empresa optou por contratar profissionais mais experientes e qualificados e atualizar o manual da oficina que continha os padrões de cada veículo. Após a segunda aplicação da metodologia FMEA, verificou-se a importância da presença do geometrista na realização dos testes junto ao cliente, quando possível, bem como a necessidade de verificação e aferição da máquina de geometria.

A metodologia GUT foi aplicada para definir as ações prioritárias frente aos problemas identificados através da combinação dos critérios gravidade e urgência. Recomendou-se que os 
esforços fossem concentrados no acompanhamento do cliente, quando possível, durante os testes do veículo após a prestação do serviço, pois, os índices de retrabalho podem ser diminuídos quando o geometrista é responsabilizado pela realização dos testes no veículo, (após o serviço prestado e na companhia/presença do cliente). O principal problema elencado pela metodologia GUT foi a aferição do equipamento de geometria. Diante disso, recomendou-se que a organização agendasse uma verificação quinzenal e se necessária a aferição semanal, realizada pelo próprio operador. De acordo com a empresa, após a aplicação das medidas corretivas o índice de retrabalho teve uma redução de sessenta e sete por cento comprovando a eficácia da aplicação das metodologias de controle FMEA e GUT na prestação do serviço de geometria a laser.

\section{REFERÊNCIAS}

ABRAPNEUS/SICOP. Cartilha de Geometria de Suspensão 2009. Disponível em: www.abrapneus.com.br/documentos/ cartilha_0001.pdf Acesso em: 16 out. 2011.

BEHR, A.; MORO, E. L. da S.; ESTABEL, L. B. Gestão da biblioteca escolar: metodologias, enfoques e aplicação de ferramentas de gestão e serviços de biblioteca. Ci. Inf. [online]. 2008, vol.37, n.2, pp. 32-42. ISSN 0100-1965.

CUNHA, F.. [et. al]. Gestão do conhecimento aplicada a segurança empresarial. Projeto final, (Especialização em Engenharia de Produção) - Programa de Pós-graduação em Engenharia de Produção. Rio de Janeiro, UFRJ/ COPPE, 2010.

FERNANDES, J. M. R. And REBELATO, M. G. Proposta de um método para integração entre QFD e FMEA. Gest. Prod. [online]. 2006, vol.13, n.2, pp. 245-259. ISSN 0104-530X.

FERNANDES, J. M. Uma proposta de integração entre métodos para o planejamento e controle da qualidade baseada no FMEA. Dissertação (Mestrado em Engenharia de Produção e Sistemas) - Programa de Pós-Graduação em Engenharia de Produção e Sistemas, Pontifícia Universidade Católica do Paraná, Curitiba. 2005.

HELMAN, H.; ANDERY, P. R. P. Análise de falhas (aplicação dos métodos de FMEA e FTA). Belo Horizonte: Fundação Christiano Ottoni, 1995.
NASCIMENTO. E. A. [et. al.]. A aplicação do MASP/PDCA em uma empresa de aviação voltada para o aumento da disponibilidade de helicópteros, modelo SIKORSKY S76C+, em operações off-shore. In: VI Congresso de Excelência em Gestão... Anais, Niterói - RJ, 2010.

OLIVEIRA, D. P. R. Planejamento Estratégico: conceitos, metodologia e práticas. 13. ed. São Paulo: Atlas, 1999.

PUENTE, J.; PINO, R.; PRIORE, P.; FOUENTE, D de $L$. A decision support system for applying failure mode and effects analysis. International Journal of Quality \& Reliability Management, Bradford, v. 19, n. 2, p. 137-151, 2002.

ROSA, L. C. da; GARRAFA, M. Análise dos modos de falha e efeitos na otimização dos fatores de produção no cultivo agrícola: subprocesso colheita da canola. Gest. Prod. [online]. 2009, vol.16, n.1, pp. 63-73. ISSN 0104-530X.

STAMATIS, D. H. Failure Mode and Effect Analysis: FMEA from theory to execution. 2. ed. ASQC, Milwaukee: Quality Press, 2003.

ZAMBRANO, T. F. and MARTINS, M. F. Utilização do método FMEA para avaliação do risco ambiental. Gest. Prod. [online]. 2007, vol.14, n.2, pp. 295-309. ISSN 0104-530X. 